\title{
An Efficient Addressing Scheme for Flexible IP Address
}

\author{
Shihao Liu \\ Computer Network Information \\ Center, Chinese Academy of Sciences \\ and University of Chinese Academy \\ of Sciences \\ liushihao@cnic.cn \\ Yihao Jia \\ Network Technology Laboratory, \\ Huawei Technologies Co., Ltd \\ jiayihao@huawei.com
}

\author{
Wanming Luo \\ Computer Network Information \\ Center, Chinese Academy of Sciences \\ lwm@cnic.cn \\ Zhe Chen \\ Network Technology Laboratory, \\ Huawei Technologies Co., Ltd \\ chenzhe17@huawei.com
}

Xu Zhou

Computer Network Information

Center, Chinese Academy of Sciences

zhouxu@cnic.cn

\author{
Sheng Jiang \\ Network Technology Laboratory, \\ Huawei Technologies Co., Ltd \\ jiangsheng@huawei.com
}

\begin{abstract}
Along with the popularization and adoption of IP in various emerging scenarios, challenges also arise with the ossified address structures. The reason is that conventional IP address is designed with fixed length and lacking extensibility, while the demand for IP varies greatly in different scenarios. Flexible IP (FlexIP), as a variablelength IP address, proactively makes address structure flexible enough to adapt to various network cases and solves the problem of low transmission efficiency faced by current IP addresses. However, due to the variable length of FlexIP, the conventional routing addressing scheme is not suitable for it. In this paper, we propose a new Bloom filter addressing scheme suitable for FlexIP address. We use controllable prefix extension to limit the prefix distribution of FlexIP, and use one-hashing to improve the computational overhead of the Bloom filter. Simulations show that the addressing scheme we proposed is more suitable for FlexIP than other schemes, and has better query efficiency.
\end{abstract}

\section{CCS CONCEPTS}

- Networks $\rightarrow$ Network protocols; Network layer protocols.

\section{KEYWORDS}

Flexible IP, Bloom filter, IP lookup, routing addressing

\section{ACM Reference Format:}

Shihao Liu, Wanming Luo, Xu Zhou, Yihao Jia, Zhe Chen, and Sheng Jiang. 2021. An Efficient Addressing Scheme for Flexible IP Address. In 2021 2nd International Conference on Control, Robotics and Intelligent System (CCRIS'21), August 20-22, 2021, Qingdao, China. ACM, New York, NY, USA, 6 pages. https://doi.org/10.1145/3483845.3483865

\footnotetext{
Permission to make digital or hard copies of all or part of this work for personal or classroom use is granted without fee provided that copies are not made or distributed for profit or commercial advantage and that copies bear this notice and the full citation on the first page. Copyrights for components of this work owned by others than ACM must be honored. Abstracting with credit is permitted. To copy otherwise, or republish, to post on servers or to redistribute to lists, requires prior specific permission and/or a fee. Request permissions from permissions@acm.org.

CCRIS'21, August 20-22, 2021, Qingdao, China

(C) 2021 Association for Computing Machinery.

ACM ISBN 978-1-4503-9045-3/21/08 . .\$15.00

https://doi.org/10.1145/3483845.3483865
}

\section{INTRODUCTION}

IP addressing has been the core issue of the Internet since the Internet was invented. With the explosive growth and dramatic development of Internet, more and more new application requirements and scenarios are emerging and the current IP address system is facing more and more problems. On the one hand, the network is rapidly expanding towards the Internet of Things (IoT), the demands for address length in different scenarios can vary drastically. AS IoT devices are limited by hardware and wireless links, a short address or device ID is more preferred than an IPv6 address for efficiency. On the other hand, the continuous emergence of emerging application requirements poses new challenges to the network, and communication subjects should be able to communicate with each other as terminals. However, IP address is the only address identification in the current data network. Although IPv6 theoretically has enough addresses, the actual address assignment efficiency makes it far less than that.

The root of all aforementioned problems lies in that IP address, either in IPv4 or in IPv6, has been designed with fixed length and thus lacks flexibility. This kind of design greatly constraints the evolvability and compatibility of the address space. The emergence of Flexible IP (FlexIP) [1] has solved this problem well. FlexIP has variable length addresses and a theoretically unlimited number of addresses. Network devices can establish a unified routing forwarding publication for addresses of different lengths, and different network addresses will coexist in data messages. A network device determines the next hop of a data message by routing table lookup operations based on addresses of any length. In IPv4/6, all addresses are stored in the routing information database with the same length, and routers can achieve efficient mask matching and addressing. However, the FlexIP address maybe with any length. If the router still stores all routing entries uniformly with the longest address length, it will pose a great challenge to storage space and lookup performance, making the current routing addressing method unacceptable.

Traditional IP addressing schemes usually include Trie-based route lookup scheme [2], [3], and hardware-based route lookup scheme [4]-[6]. The Trie-based routing search scheme has a simple structure and is easy to implement, but each search requires a large amount of memory access, and the length of the IP address greatly restricts its query performance, so it is not suitable for FlexIP 
addressing. The hardware-based route lookup scheme is limited by high cost and power consumption, and it is also not considered. Bloom filter [7] as a streamlined and efficient Hash solution has been widely used in various network scenarios. Sarang.D first applied the Bloom filter to route lookup [8]. Although this scheme has made some progress compared with the traditional route lookup scheme, the number of Bloom filters depends on the number of different IP address prefixes lengths. Lim proposed to use one bloom filter to find the longest matching prefix length [9]. Haoyu.S proposed a distributed Bloom filter addressing scheme [10]. Some Bloom filter-based IP lookup algorithms can also be found in the literature [11]-[13]. However, the structure of previous Bloom filter-based schemes is too complicated to be suitable for FlexIP addressing.

Main contributions. In this paper, we propose a new efficient addressing scheme suitable for FlexIP address, which fills the gap of current research. We use Controlled Prefix Expansion (CPE) to limit the prefix length of FlexIP, which reduces the number of Bloom filters and reduces the impact of the false positive. Simultaneously, we reduce the number of hash functions in each Bloom filter to one through one-hashing method, which greatly reduces the computational cost caused by CPE. Further, we analyze its excellent performance in theory. Simulations have proved that our scheme has better query efficiency than other schemes for FlexIP addressing.

\section{RELATED WORK}

\subsection{Flexible IP and Multi-Entrance-Trie}

Due to the explosive growth and rapid development of the Internet, the current IP address system faces some serious problems. In order to solve the above problems, Flexible IP (FlexIP) was designed. Specific information about FlexIP can be obtained from [1] and [14].

Multi-Entrance-Trie (METrie) was first proposed for FlexIP addressing. METrie uses a multi-routing table storage method, and creatively expands the target address, which has inspired a lot of our work. However, the structure of METrie is too complicated, and especially the dynamic update operation requires a lot of pruning and expansion and frequent memory access. The addressing scheme based on the Bloom filter proposed in this paper is more streamlined in structure, and the update operation is easier. It is proved in the simulations that it can query more efficiently than METrie.

\subsection{Bloom Filter and Variations}

The Standard Bloom Filter (SBF) is a data structure that uses a bit vector of length $m$ and $k$ different mutually independent hash functions to represent a set $S$ with $n$ elements. The false positive probability of SBF, $f_{S B F}$ is

$$
f_{S B F} \approx\left(1-e^{-\frac{n k}{m}}\right)^{k}
$$

In the optimal case, we can get the following relationship of the false positive probability

$$
k=\frac{m}{2} \ln 2 .
$$

$$
f_{S B F}=\left(\frac{1}{2}\right)^{k} .
$$

Jianyuan.L's One-Hashing Bloom Filter (OHBF) [16] proposes a new algorithm to solve the calculation overhead of the Bloom filter. This method only needs one base hash function plus a few simple operations to implement a Bloom filter, and the simulations prove that the false positive probability of OHBF is very close to SBF and has good performance. The improvement of $\mathrm{OHBF}$ has a great effect on the addressing scheme of FlexIP and is adopted by us.

\section{DESIGN OF ADDRESSING SCHEME}

The addressing scheme based on the Bloom filter is essentially a hash-based scheme, that is, we use all prefixes to build hash tables in off-chip memory and store the next hop information. For IP lookup, the Bloom filter in on-chip memory can be used to quickly find the possible matching prefix and check it in the off-chip hash tables. Figure 1 (a) shows the configuration of the traditional Bloom filterbased scheme (TBF-based scheme). First, the prefixes are grouped based on their prefix lengths, and each group corresponds to one Bloom filter, which is used to store all prefixes of that length. Then, all possible prefixes of the IP address to be queried are input in parallel into all Bloom filters, and the Bloom filter returns whether the prefix exists. But at this time Bloom filters may have the probability of false positives. In order to verify whether it is a true match, check off-chip hash tables from the longest prefix according to the longest match principle. If this prefix exists in the hash tables, get the next hop, otherwise, continue to check the next prefix until the match is successful or the default forwarding is obtained.

Although the TBF-based scheme is simple to implement, it still faces some challenges. The first problem is the impact of off-chip memory access. When the TBF-based scheme is applied to IPv6 addressing, a 128-bit address means that 128 Bloom filters are required. If multiple Bloom filters are false positives, the number of checking off-chip hash tables will become unacceptable. In the worst case, it needs to check off-chip memory 128 times. Although the possibility of this is very low, it should still be avoided. Compared with IPv6 addressing, the TBF-based scheme is more difficult to complete FlexIP addressing with flexible address length. Another problem with the TBF-based scheme is the hash function. To ensure the reliability of Bloom Filters queries, the number of hash functions must increase as the entries in the RIB increase, which will increase the computational of Bloom filters, and it is difficult to find a large number of independent hash functions with excellent performance.

In order to solve the shortcomings in the TBF-based scheme, we proposed a Bloom filter addressing scheme combining CPE [15] and one-hashing [16], it can be referred to as COBF-based scheme for short. In order to reduce the number of off-chip memory access in the worst case as much as possible, we must reduce the number of Bloom filters. Since different Bloom filters correspond to prefixes of different lengths, the number of prefix lengths needs to be limited. CPE can simply extend a prefix of length $L$ to a set of prefixes of length $W(L<W)$. Obviously, CPE will cause a large increase in the number of prefixes in the RIB. To ensure the upper limit of the false positive probability of Bloom filters, it is necessary to increase the number of hash functions. To solve this problem caused by $\mathrm{CPE}$, we adopted the one-hashing method to optimize Bloom filters. 


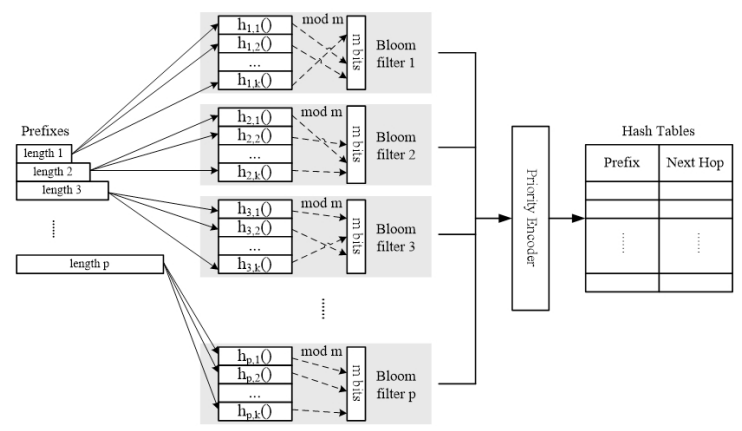

(a) TBF-based scheme

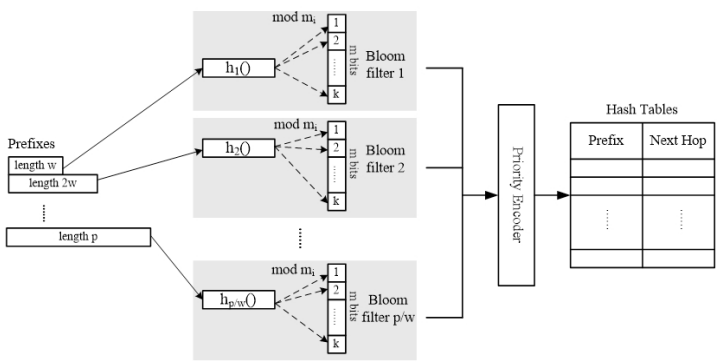

(b) COBF-based scheme

Figure 1: Configuration of TBF-based scheme and COBF-based scheme

This method can replace $k$ hash functions with one hash function and multiple simple modular operations, the computation cost is effectively reduced to $1 / k$.

Figure 1 (b) illustrates the configuration of the COBF-based scheme. We divide all the prefixes into several continuous disjoint intervals. The difference between the length of the longest prefix and the length of the shortest prefix in the interval is called the interval width, $w$. Each interval is extended to the upper limit of the interval through CPE so that each interval only contains the prefixes with the same longest length. CPE makes our prefix set only contain prefixes of length $w, 2 w, \ldots, p$, and reduces the number of Bloom filters from $p$ to $p / w$. Of course, the specific number of Bloom filters used depends on the selected interval width, $w$, which is limited by the memory size. Further, we divide the bit vector of each Bloom filter into $k$ partitions, the number of partitions is equal to the number of hash functions in the SBF. We select $k$ consecutive prime numbers as the length of $k$ partitions and make the sum of the lengths of $k$ partitions as close as possible to the length of the bit vector. Then, each Bloom filter only needs one hash function and $k$ modular operations to complete a query or update. For IP lookup, similar to the TBF-based scheme, all possible prefixes of the IP address are input in parallel to all Bloom filters for search. According to the feedback result of the Bloom filters, the off-chip hash tables are checked by the longest match principle until the next hop is obtained.

Dynamic update. To ensure the completion of the prefix update operation, we need to maintain a set of counters to record more information. Each bit of the bit vector of the Bloom filter is respectively associated with a counter, and the prefix insertion or deletion is completed by adding and subtracting the corresponding $k$ counters. In practical applications, the update operation of the prefix is far less than the query operation of the prefix, so we can put the counters in the off-chip memory, which can reduce the use of embedded memory.

\section{THEORETICAL ANALYSIS}

\subsection{Addressing Performance}

The performance of the COBF-based scheme mainly depends on the number of checking off-chip hash tables, and the reason for checking the hash tables multiple times is the false positive of the
Bloom filters. In practical applications, we usually set the bit vector of each Bloom filter to the same size and divide it into the same partitions. Although the number of prefixes stored in each Bloom filter is different, for the convenience of analysis, we assume that each Bloom filter stores $n$ prefixes and its bit vector is $m$ bits. The bit vector of each Bloom filter is divided into $k$ partitions, and the length of each partition is $m_{i}, 0<i \leq k$. Therefore, every Bloom filter has the same false positive probability $f \approx\left(1-\sqrt[k]{\prod_{i=1}^{k} e^{-\frac{n}{m_{i}}}}\right)^{k}$.

The number of Bloom filters used depends on the number of different prefix lengths and the extended interval width. Assuming that the number of prefixes of different lengths of FlexIP is $P$ and the interval width is $w$, then the number of Bloom filters that need to be used is $P / w$. Obviously, if multiple Bloom filters show false positives, the number of checks on the off-chip hash tables will inevitably increase. For a prefix of length $l$, all false positives of Bloom filter whose prefix length is greater than $l$ will affect its query. Then, the maximum number of additional checking off-chip hash tables for a search is

$$
E_{\text {max }}=\frac{P}{w} f .
$$

On average, the upper limit of the number of off-chip hash tables to be checked for a successful search is

$$
E_{\text {avg }} \leq E_{\text {max }}+1=\frac{P}{w} f+1 .
$$

The worst case is that all Bloom filters show false positives during one search. So, the number of off-chip hash tables that need to be checked is

$$
E_{\text {worst }}=\frac{P}{w}+1
$$

\subsection{Memory Occupancy}

We only discuss the on-chip memory usage. Assuming that the COBF-based scheme needs to meet the addressing needs of $N$ FlexIPs. After the CPE processing, the number of prefixes to be stored, $N_{C P E}$ is

$$
N_{C P E} \approx \sum_{i=1}^{P} \frac{N}{P} \cdot 2^{w-l \bmod w} \approx \frac{P}{w} \cdot n .
$$

Assuming that the COBF-based scheme needs to occupy $M$ bits of on-chip memory. Since under ideal conditions, $f \approx f_{S B F} \approx$ 
Table 1: Simulation database used in this paper

\begin{tabular}{lll}
\hline Database & Prefixes & Prefix Length Distributions \\
\hline Database 1 & 100,000 & evenly distribution within 128-bit \\
Database 2 & 100,000 & {$[13,16]: 33.33 \%,[29,32]: 33.33 \%,[61,64]: 33.33 \%$ and length within 128 -bit } \\
Database 3 & 122,416 & $/ 48: 45.90 \%, / 32: 13.49 \%, / 44: 9.40 \%, / 40: 8.01 \%, / 36: 3.76 \%$, the others:19.44\% \\
\hline
\end{tabular}
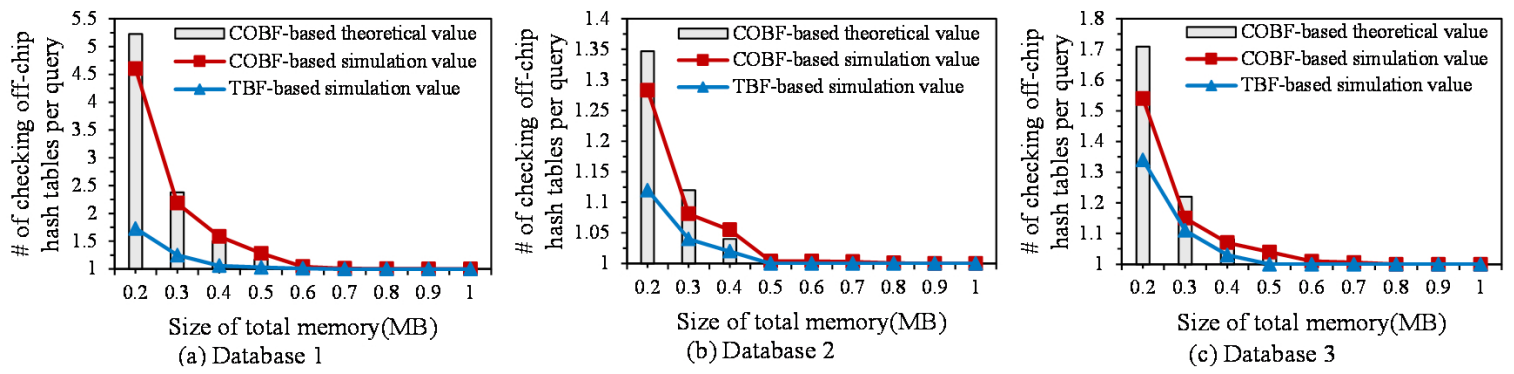

Figure 2: The average number of checking off-chip hash tables per query, for variable $M$.

$\left(1-e^{\frac{-n k}{m}}\right)^{k}$, then

$$
M \approx \frac{P}{w} \cdot m \approx \frac{P}{w} \cdot\left(-\frac{k n}{\ln \left(1-f^{\frac{1}{k}}\right)}\right) .
$$

Let $\frac{d f(k)}{d k}=0$, then

$$
k_{\text {min }}=(\ln 2)\left(\frac{m}{n}\right)=(\ln 2)\left(\frac{m}{\sum_{l=1}^{w}\left(\frac{N}{P} \cdot 2^{w-l}\right)}\right) .
$$

Under certain addressing scale and addressing performance, $k_{\text {min }}$ makes the minimum on-chip memory, $M_{\min }$ occupied by COBF-based scheme as

$$
M_{\text {min }}=-\frac{p}{w} \cdot \frac{n \ln f_{\text {min }}}{(\ln 2)^{2}}=-\frac{p}{w} \cdot \frac{\sum_{l=1}^{w}\left(\frac{N}{P} \cdot 2^{w-l}\right) \ln f_{\text {min }}}{(\ln 2)^{2}} .
$$

\section{PERFORMANCE EVALUATION}

We conducted some simulations to verify the effectiveness of the COBF-based scheme. All simulations were performed on a standard off laptop computer, with an Intel(R) Core i7-8750H CPU @2.20GHz and 16GB RAM.

We set up three sets of different routing tables as the database for the simulation. Since FlexIP is still in the research stage, there is no real routing data at present, so we randomly generated 100, 000 FlexIP addresses within 128 bits as the simulations database 1, and their prefixes lengths are evenly distributed. For comparison, we generated 100,000 FlexIP addresses with uneven prefixes length distribution as database 2. In addition, we also used a real IPv6 BGP routing table from $h t t p: / / b g p . p o t a r o o . n e t$, which has a more uneven distribution of prefix lengths. The database is shown in Table 1.

In the simulation, we compared the COBF-based scheme with the other two schemes, namely METrie and TBF-based scheme. METrie is the first scheme proposed specifically for FlexIP addressing, and TBF-based is the basis of the COBF-based solution. This can more comprehensively show the FlexIP addressing performance of the COBF-based scheme.

\subsection{The Impact of False Positives}

We first evaluated the impact of false positives on the COBF-based scheme, because this will directly affect the performance of addressing, which is mainly reflected in the number of checking off-chip hash tables per query. In the simulation, we set $N=100,000, w=$ $4, k$ is the optimal value calculated by (2), and observe the influence of $M$ on COBF-based, we use (5) to calculate the theoretical upper limit of $E_{a v g}$, and compare the results with TBF-based.

Figure 2 shows that the COBF-based scheme meets the theoretical upper limit under various configurations, which proves the correctness of the scheme. We can also see that the distribution of prefixes length has a great influence on addressing, the completely uniform distribution in figure 2 (a) is the worst addressing case. This is mainly due to the scattered prefixes increasing the number of Bloom filters. In addition, due to the increase in the number of prefixes caused by CPE, the total memory used by the COBF-based scheme is slightly higher than that of the TBF-based scheme, but this is still acceptable. The difference in memory usage of these two schemes with different databases is about $0.1 \mathrm{MB}$ when the performance is close to 1 off-chip hash tables check per query. The COBF-based scheme can use moderate memory to obtain a query efficiency much higher than that of the TBF-based scheme, which will be proved in the next simulation.

\subsection{Addressing Performance}

In order to verify the addressing performance of the COBF-based scheme in practical applications, we observed the addressing speed of FlexIP through simulations and compared it with the METrie scheme and the TBF-based scheme. For the METrie scheme, we have designed 8 entry nodes for it, and the corresponding length is 16bits, 32bits, ..., 128bits. For TBF-based scheme and COBF-based 


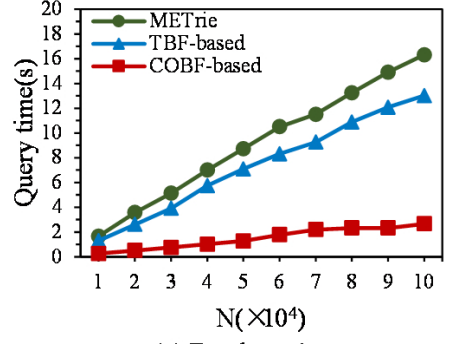

(a) Database 1

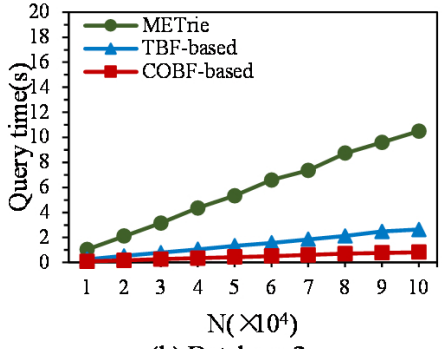

(b) Database 2

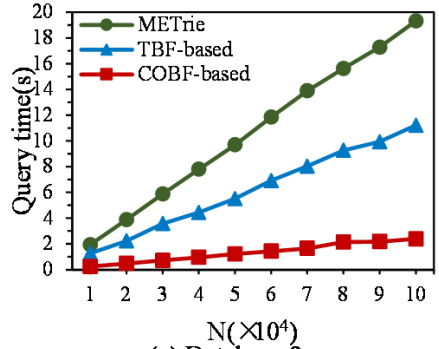

(c) Database 3

Figure 3: Comparison of the query time of the three schemes.

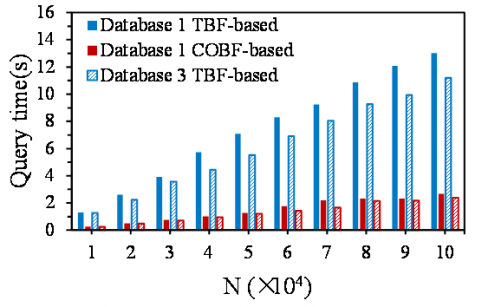

(a) Changing $\mathrm{N}(\mathrm{M}=1 \mathrm{MB}, \mathrm{k}=8)$

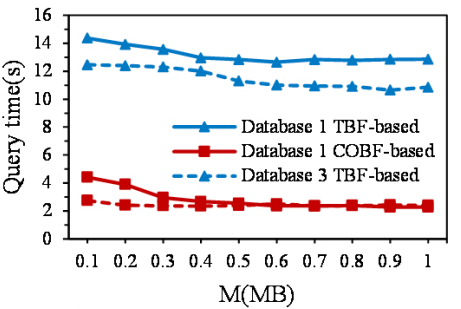

(b) Changing $M(N=100000, k=8)$

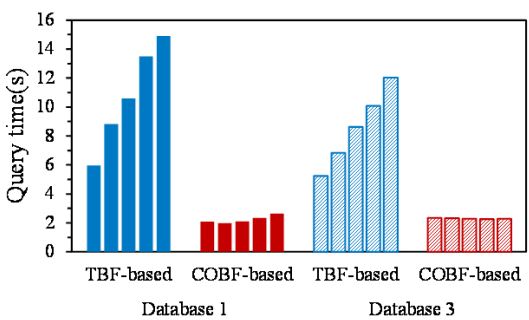

(c) Changing $k$ from 2 to $10(\mathrm{~N}=100000, \mathrm{M}=1 \mathrm{MB})$

Figure 4: Comparison of COBF-based and METrie memory accesses.

scheme, we set $M=4 M b, k=8, w=4$. For all schemes, we change the size of $N$ and test it in three databases.

Figure 3 shows the query time of the three schemes under different percentages of the query set. We can see that the COBF-based scheme has the minimum query time, significantly lower than TBFbased scheme and METrie scheme. With the increase of the query set, the query time of the three schemes becomes larger, and the query time of the METrie scheme and the TBF-based scheme increases almost linearly while the COBF-based query time is less affected.

Furthermore, in order to prove the effectiveness of $\mathrm{CPE}$ and onehashing adopted by COBF-based scheme, we tested and compared the query time of COBF-based scheme and TBF-based scheme under different configurations. The results are shown in Figure 4. COBFbased query speed is significantly better than TBF-based in various configurations, the main reason is that $\mathrm{CPE}$ reduces the use of the Bloom filter. Moreover, when the value of $k$ changes, the calculation cost of the TBF-based scheme increases due to the increase of hash functions, which makes the query time sharply increased. The COBF-basd scheme benefits from the optimization of one-hashing, and the query time is almost unaffected. It is worth noting that with the increase of total memory, $M$, the query time of both decreases first and then tends to be stable. As the total memory increases until the false positive probability is close to 0 , the addressing performance gradually reaches the optimum, and at this time, the value of $M$ should also be the memory choice of the addressing scheme.

\section{CONCLUSION}

In order to meet the efficient addressing requirements of FlexIP, we designed a COBF-based scheme based on the TBF-based scheme.
This scheme reduces the number of Bloom filters used through $\mathrm{CPE}$, which effectively reduces the impact of false positives, and uses one-hashing to reduce the computational cost of Bloom filters. The COBF-based scheme has good scalability and simple update operations. Simulations have shown that this scheme is close to one off-chip hash tables check per query, and it is better than existing addressing schemes in query time.

\section{ACKNOWLEDGMENTS}

This work is supported in part by the National Key R\&D Program of China under Grant 2018YFB1800100, and the Industrial Internet edge computing basic standards and experimental verification project of industrial Internet innovation and development program of the Ministry of industry and information technology in 2018, and Open Research of Zhejiang Lab under Grant No.2021LC0AB03.

\section{REFERENCES}

[1] Ren, Shoushou, et al. "Routing and Addressing with Length Variable IP Address." Proceedings of the ACM SIGCOMM 2019 Workshop on Networking for Emerging Applications and Technologies, 2019, pp. 43-48.

[2] H. Asai and Y. Ohara, "Poptrie: A compressed trie with population count for fast and scalable software IP routing table lookup," in Proc. ACM SIGCOMM, 2015, pp. $57-70$.

[3] Rétvári, Gábor, et al. "Compressing IP Forwarding Tables: Towards Entropy Bounds and Beyond." IEEE ACM Transactions on Networking, vol. 24, no. 1, 2016, pp. 149-162.

[4] Huang, Jhih-Yu, and Pi-Chung Wang. "TCAM-Based IP Address Lookup Using Longest Suffix Split." IEEE ACM Transactions on Networking, vol. 26, no. 2, 2018, pp. 976-989.

[5] Wenjun, Li, and Li Xianfeng. TCAM-Based High-Speed and Low-Power IP Routing Table Lookup Method. 2015.

[6] Sun, Yan, et al. "Using TCAM Efficiently for IP Route Lookup." 2011 IEEE Consumer Communications and Networking Conference (CCNC), 2011, pp. 816-817.

[7] Bloom, Burton H. "Space/Time Trade-Offs in Hash Coding with Allowable Errors." Communications of The ACM, vol. 13, no. 7, 1970, pp. 422-426. 
[8] Dharmapurikar, Sarang, et al. "Longest Prefix Matching Using Bloom Filters." IEEE ACM Transactions on Networking, vol. 14, no. 2, 2006, pp. 397-409.

[9] Lim, Hyesook, et al. "On Adding Bloom Filters to Longest Prefix Matching Algorithms." IEEE Transactions on Computers, vol. 63, no. 2, 2014, pp. 411-423.

[10] Song, H., et al. "IPv6 Lookups Using Distributed and Load Balanced Bloom Filters for 100Gbps Core Router Line Cards." IEEE INFOCOM 2009, 2009, pp. 2518-2526.

[11] J. H. Mun and H. Lim, "New Approach for Efficient IP Address Lookup Using a Bloom Filter in Trie-Based Algorithms," in IEEE Transactions on Computers, vol. 65, no. 5, pp. 1558-1565, 1 May 2016, doi: 10.1109/TC.2015.2444850.

[12] H. Lim, J. Lee and C. Yim, "Complement Bloom Filter for Identifying True Positiveness of a Bloom Filter," in IEEE Communications Letters, vol. 19, no. 11, pp. 1905-1908, Nov. 2015, doi: 10.1109/LCOMM.2015.2478462.
[13] Jungwon Lee, Miran Shim, and Hyesook Lim. 2016. Name prefix matching using bloom filter pre-searching for content centric network. J. Netw. Comput. Appl. 65, C (April 2016), 36-47. DOI:https://doi.org/10.1016/j.jnca.2016.02.008.

[14] Chen, Zhe, et al. "NEW IP Framework and Protocol for Future Applications." NOMS 2020 - 2020 IEEE/IFIP Network Operations and Management Symposium, 2020, pp. $1-5$.

[15] Srinivasan, V., and George Varghese. "Faster IP Lookups Using Controlled Prefix Expansion." Proceedings of the 1998 ACM SIGMETRICS Joint International Conference on Measurement and Modeling of Computer Systems, vol. 26, no. 1, 1998, pp. 1-10.

[16] Lu, Jianyuan, et al. "One-Hashing Bloom Filter." 2015 IEEE 23rd International Symposium on Quality of Service (IWQoS), 2015, pp. 289-298. 